\title{
TOMOGRAPHY
}

\section{Movies of evolving objects from a single laser shot}

Tomography is typically associated with the generation of cross-sectional images of a stationary object by measuring the absorption of radiation beams incident at various angles on the object. Now, Zhengyan Li and co-workers at the University of Texas in Austin, USA, report an adaption of tomography that uses a single laser pulse to generate movies of evolving 'light-velocity objects' - transient perturbations in the refractive index induced by pulses of light or high-velocity particles (Nature Commun. 5, 3085; 2014). These tomographic movies, the frames of which are essentially slices in both space and time, reveal the spatiotemporal evolution of the object and can be used to examine phenomena as diverse as rogue waves, plasma wakefield acceleration and filament formation in gases.

Previously developed techniques can generate pseudo-movies of such objects by stacking snapshots obtained using different pulses in a time sequence. However, they are based on the assumption that each pulse evolves identically - something that does not hold for objects that evolve stochastically, propagate in turbulent media or fluctuate from shot to shot.

In contrast, the approach introduced by Li et al., which they call frequency-domain tomography, employs a combination of four-wave mixing, second-harmonic generation and chirping in a nonlinearcrystal sandwich (HZF4 glass/ $\beta$-barium oxide/HZF4 glass) to generate multiple probe pulses at various angles of incidence
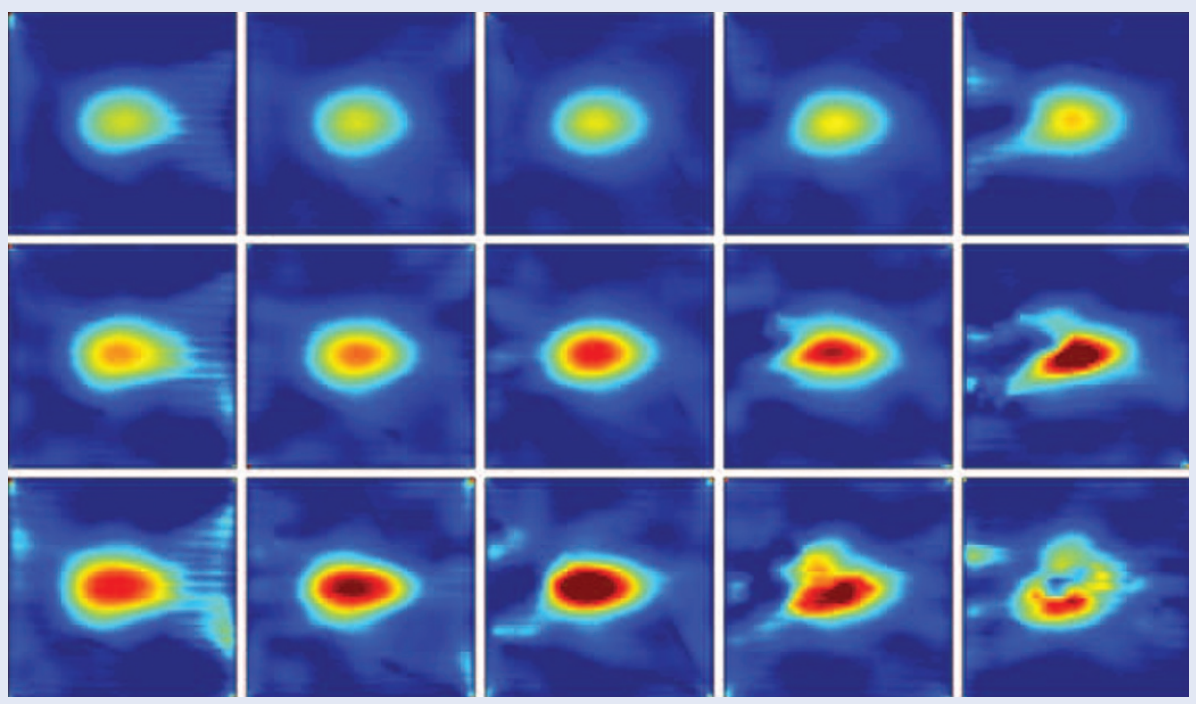

in a single step. As these pulses propagate through the medium they interact with the evolving object, and are imprinted with a 'phase streak' via cross-phase modulation in the process. The pulses are then simultaneously detected by a chargedcoupled device detector in a spectrometer. Finally, a modified tomography algorithm is used to generate movie frames of the object moving through the medium.

The researchers demonstrated the effectiveness of the technique by using it to produce movies of a laser pulse propagating through a transparent Kerr medium. The movies revealed diffraction, self-focusing and filamentation of the pulse and were in good agreement with simulations based on the nonlinear Schrödinger equation.

The group leader, Michael Downer, told Nature Photonics that the team is currently investigating using the technique "to visualize plasma wakefield accelerator structures driven by petawatt laser pulses and charged particle bunches." He anticipates that "tomographic movies will open these structures to direct, immediate view in the laboratory and facilitate understanding, optimization and scaling of table-top accelerators and the future X-ray free-electron lasers that they will drive."

SIMON PLEASANTS

\section{OPTICAL PHYSICS}

\section{Extending filamentation}

The introduction of a dressing beam to extend the reach of filaments promises a wealth of new applications that extend beyond the laboratory scale.

\section{Günter Steinmeyer and Carsten Brée}

F emtosecond filamentation is

an appealing self-organization phenomenon in optical physics that has been awaiting practical applications for a long time. First described some 20 years ago $^{1}$, the collapse of the spatial beam profile of a femtosecond laser beam may lead to the formation of metre-long plasma channels in atmospheric air. These plasma channels resemble those generated by electric discharges but are absolutely straight, without any bends or turns. Despite the undisputed beauty of this phenomenon, femtosecond filamentation has led to only a limited number of applications to date (for example, pulse self-compression ${ }^{2}$ ). One critical issue hindering the development of many other attractive applications is the restricted length of single filaments.

Writing in Nature Photonics, Maik Scheller and colleagues describe the use of dressing laser beams as a promising new approach for generating extended 